\title{
Bacterial flora associated with udder cleft dermatitis in Dutch dairy cows
}

\author{
E. van Engelen, ${ }^{1 *}$ (다 T. Dijkstra, ${ }^{1}$ N. M. Meertens, ${ }^{1}$ (i) and T. van Werven ${ }^{2,3}$ (i) \\ ${ }^{1}$ Royal GD, 7400 AA Deventer, the Netherlands \\ ${ }^{2}$ Department of Farm Animal Health, Faculty of Veterinary Medicine, Utrecht University, 3584 CL Utrecht, the Netherlands \\ ${ }^{3}$ University Farm Animal Practice, 3484 LZ Harmelen, the Netherlands
}

\begin{abstract}
Udder cleft dermatitis (UCD) or udder sores is a skin lesion, characteristically located around the anterior junction between the udder and abdomen of dairy cows. It is a worldwide problem in dairy herds with a large effect on animal welfare. The etiology and possible infectious origins of UCD are largely unknown; however, specific bacterial or parasitic causes are suggested in the literature. Therefore the aim of this study was to investigate the possible bacteriological, mycological, or parasitic involvement in clinically scored UCD lesions. Bacteriological culture was performed on subcutaneous tissue samples taken postmortem at a depth of 5 to 10 $\mathrm{mm}$ of 87 mild or severe UCD lesions or from the same place in healthy cows. Fungal culture was performed on a subset of 22 subcutaneous tissue samples of severe UCD postmortem. To investigate the superficial flora, swabs were taken from normal skin or skin lesions of 15 live animals equally divided over 3 groups: healthy skin or mild and severe UCD lesions. Histopathology, to describe and classify the lesions and to assess the presence of mites, fungi, or bacteria, was performed on 128 tissue samples, taken separately. In severe UCD lesions, Trueperella pyogenes and Bacteroides pyogenes were more frequently present in deep tissue layers and in superficial layers, compared with the same layers in mild UCD lesions or healthy skin. Culturing and histopathology indicated no sign of involvement of treponemes, Staphylococcus aureus, Escherichia coli, fungi, or mites in the UCD lesions. Histopathological examination showed that the majority of the lesions were characterized by chronic aspecific inflammation. Severe UCD lesions more frequently showed chronic active inflammation on histopathology, compared with mild UCD lesions. Due to the cross-sectional character
\end{abstract}

Received February 24, 2020.

Accepted August 25, 2020.

*Corresponding author: e.v.engelen@gdanimalhealth.com of this study, it is difficult to differentiate cause and effect; however, future preventive and curative measures against UCD should take into account the chronic and anaerobic nature of this illness.

Key words: udder cleft dermatitis, bacteriology, dairy cow, histopathology

\section{INTRODUCTION}

Udder cleft dermatitis (UCD) has been described as an exudative and ulcerative dermatitis localized between the front quarters of the udder or at the anterior junction between the udder and abdomen of dairy cows. Macroscopically the lesions can be categorized as mild or severe UCD (Bouma et al., 2016). However, in severe cases, the infection can spread and cause embolic disease, leading to death (Millar et al., 2017). In 2019 in the Netherlands, postmortem examination revealed 20 cases of embolic pneumonia as the cause of death due to thrombophlebitis of milk veins as a result of ulcerative UCD (Scherpenzeel et al., 2020). In several countries UCD has been reported with between-herd prevalence varying between 0 and $43 \%$. (Olde Riekerink et al., 2014; Bouma et al., 2016; Ekman et al., 2018). Most of these studies were set up to calculate the prevalence (Olde Riekerink et al., 2014; Ekman et al., 2018) or the incidence (Bouma et al., 2016) of UCD. Several cow-based factors appear to play a role in the occurrence of UCD, such as a deep udder, large fore quarters, a small angle between the udder and the abdominal wall, DIM, high milk yield, lameness, and parity, as well as farm-based factors (Olde Riekerink et al., 2014; Ekman et al., 2018). However, involvement of an infectious agent cannot be ruled out. In fact, few studies have focused on the possible causative agents of UCD. Warnick et al. (2002) studied the possible involvement of Sarcoptes mites but concluded that parasitic control did not eliminate UCD lesions. Other studies suggest the involvement of treponemes, based on a positive correlation between the prevalence of UCD and digital dermatitis on farm level or detection of treponemes in udder skin or foremilk (Stamm et al., 2009; Evans et 
al., 2010; Sobhy et al., 2019). However, this was not supported by a recent study by Sorge et al. (2019), in which the authors concluded that it seemed unlikely that spirochetes play a major role in the development of UCD. Although a bacteriological cause of UCD lesions is not yet known, farmers often treat the lesions with sprays or ointments containing antimicrobial agents (van Werven et al., 2018). Presence of bacteria other than treponemes in UCD lesions was described in a recent study using a metagenomic approach (Sorge et al., 2019). Those authors assessed the presence of bacterial DNA in punch biopsies taken from lesions and control tissues. In cases of UCD, more DNA of anaerobic bacteria such as Fusobacterium, Helcococcus, Anaerococcus, Porphyromonas, Prevotella, and Trueperella was seen. However, using this approach, bacteria could not be identified at species level. Additionally, it was not possible to determine whether the bacteria had invaded the tissue or were present at the surface of the lesion. This is relevant because bacteria are not typically present in deeper tissues (Grice and Segre, 2011), and, when present there, they are more likely playing an active role in the pathogenesis. In our study, we aimed to assess bacteriological, mycological, or parasitic involvement in mild and severe UCD lesions. For this, we investigated the presence of bacteria, fungi, and parasites in the deeper and superficial layers of UCD lesions separately by culture and related this to histopathology of the lesions and the clinical scoring.

\section{MATERIALS AND METHODS}

Samples for bacteriological or fungal culture and for histopathological examination were taken from postmortem material in the slaughterhouse and from live lactating dairy cows with and without UCD lesions. Eighty-seven postmortem cows were sampled for both histopathology and deep-layer bacteriology, and 41 postmortem cows were sampled for histopathology alone. In addition, 15 live lactating cows were sampled for superficial-layer bacteriology (Table 1).

\section{Sampling}

Postmortem. Sampling of lesions of slaughtered dairy cows was performed at 3 different Dutch slaughterhouses. A total of 128 animals was sampled. From these animals, the udder skin was categorized for the presence and severity of UCD lesions, as healthy, mild, or severe, as described previously (Bouma et al., 2016). Because the numbers of cases differed per category, we had to select animals for each category to obtain sufficient sample sizes. Within each category, sampling was random. Immediately after stunning and exsanguination of the cow, samples were taken from UCD lesions or from healthy skin at the place of predilection, in the center of the anterior junction between udder and abdomen in healthy animals. A part of the skin lesion, including the transition zone to healthy skin, approximately $2 \mathrm{~cm}$ thick was excised with a scalpel, kept in sterile Petri dishes, and transported to the laboratory at a temperature of $0-8^{\circ} \mathrm{C}$. Further processing took place in a cabinet, where tissue samples were taken for both bacteriology and histopathology. Samples were taken from the transition zone between healthy skin and lesion, because we regarded this as giving the highest probability to find a causative agent and ensuring that the epidermis and dermis, and not only ulcerated or necrotic tissue, are present in the sampled tissue for histopathological examination. Per lesion site, 2 tissue samples were taken close to each other with a sterile 6-mm biopsy punch (Servoprax GmbH, Wesel, Germany) and sterile scalpel (Swann-Morton, Sheffield, UK), one sample for histopathology and one for bacteriological culture. With regard to the sample for bacteriological culture, to prevent contamination with bacteria from the environment, the superficial skin was sterilized by contact application of a heated iron for about a second before taking the tissue sample. The superficial layer was not suitable for culture due to the scalding procedure, so this part of the tissue was removed and the samples were taken from a depth of 5 to $10 \mathrm{~mm}$. Samples for bacteriological culture were stored in Portagerm tubes with transport medium

Table 1. Overview of the number of cows from which samples were taken for bacteriological culturing $(\mathrm{n}=$ $102)$ and histopathological examination $(\mathrm{n}=128)$; samples were distributed over the udder cleft dermatitis (UCD) categories healthy skin, mild UCD, or severe UCD

\begin{tabular}{lcrrr}
\hline & \multicolumn{3}{c}{ UCD category } & \\
\cline { 2 - 3 } Item & Healthy skin & Mild & Severe & Total \\
\hline Bacteriological culture & & & & \\
$\quad$ Deep-layer tissue samples, postmortem & 28 & 33 & 26 & 87 \\
$\quad$ Swab samples, living cows & 5 & 5 & 5 & 15 \\
Histopathology & 45 & 42 & 41 & 128 \\
$\quad$ Tissue samples, postmortem & 45 & & \\
\hline
\end{tabular}


(BioMérieux, Marcy-l'Étoile, France) at 0 to $8^{\circ} \mathrm{C}$, to be processed within $12 \mathrm{~h}$.

Live Cows. To investigate the superficial flora of UCD lesions, 15 swabs were taken from live animals from one commercial dairy farm, that had been included in a longitudinal UCD field study as described previously (Bouma et a., 2016). Dry swab samples were taken from the same location as sampled postmortem, transported in Portagerm tubes (BioMérieux), and processed as described for the postmortem samples. We took 5 samples from healthy skin, 5 from mild UCD lesions, and 5 from severe UCD lesions.

\section{Microbiology}

In total 102 samples were used for bacteriological culture: 87 deep tissue samples from dead animals and 15 superficial swab samples from living animals. Origins of the samples was as follows: 33 from healthy skin, 38 from mild UCD lesions, and 31 from severe UCD lesions.

Tissue samples were cultured within a day after arrival at the laboratory. They were inoculated on 3 plates: 2 sheep blood agar (SBA) plates and a Columbia sheep blood agar plate enriched with gram-negative supplement, aimed to enhance the growth of gram-negative strict anaerobic bacteria (G-N Anaerobe Supplement, Oxoid; CSGN). Inoculation was performed using a 3 -phase streaking pattern. The tissue was streaked at one section of the plate, and this material was further spread sequentially by sterile loops for further dilution of the materials at successive sections of the plate. One SBA plate and the CSGN plate were incubated under anaerobic conditions and one SBA plate under aerobic conditions. Additionally, samples were spread on Sabouraud plates to assess the presence of fungi. If fungal infection played a role in UCD, we regarded culture of the severe UCD lesions as likely to give the highest chance of culturing the fungi. Therefore, 22 samples were randomly chosen from the severe UCD category.

The SBA plates were incubated for $48 \mathrm{~h}$ at $37^{\circ} \mathrm{C}$, checked for presence of Staphylococcus aureus or Escherichia coli after $24 \mathrm{~h}$, and re-evaluated for presence of Trueperella pyogenes colonies after $48 \mathrm{~h}$. The level of growth was semiquantitatively described as no growth, only growth in the first section $=$ few, growth in both first and second section $=$ moderate, or growth in all sections $=$ abundant (Kallstrom, 2014). Colonies were identified via MALDI-TOF MS, using standard software (Bruker Daltonics, Hamburg, Germany).

The CSGN plates were incubated for $5 \mathrm{~d}$ at $37^{\circ} \mathrm{C}$ under anaerobic conditions. After $3 \mathrm{~d}$, the plates were evaluated for growth. For these cultures, all visible different colony types were taken into consideration and described with respect to morphology and abundance. After this, one colony of each type was taken for pure culture, after which isolates were stored separately. This was done for a maximum of the 10 different most abundant colony types per plate. After $5 \mathrm{~d}$ of culture, the plates were re-evaluated for additional growth. If new colony types were seen, these bacteria were also isolated. Bacteria were identified via MALDI-TOF MS using standard software (Bruker Daltonics).

\section{Histopathology}

Processing and Histochemical Stains. Tissue samples for histopathology were taken of in total 128 animals, as previously described (Table 1). The tissues were fixated in $10 \%$ neutral buffered formalin, routinely processed, and embedded in paraffin wax. Routine hematoxylin and eosin $(\mathbf{H E})$-stained $2-\mu \mathrm{m}$ sections were made and examined via light microscopy. Additionally, Wharthin-Starry staining (a silver stain for the visualization of spirochaetes), Brown and Brenn staining (a modified Gram stain for bacteria), and periodic acid Schiff staining (for the visualization of fungi and yeasts) were performed on cases with acute or subacute inflammatory lesions (Suvarna et al., 2019).

Histopathological Examination. The histopathological examination of the tissue samples was performed by a European College of Veterinary Pathology boardcertified pathologist (N. M. M.). The lesions were described using internationally recognized descriptive terminology (McGavin and Zachary, 2007). Based on the predominant histopathological features, the 128 cases were categorized as follows: normal skin (no abnormalities or only minimal inflammatory changes, which can be found in healthy, non-lesional animals), acute lesions [showing ulceration or mainly neutrophilic inflammation (Figures 1 and 2), without fibrosis], subacute lesions [showing mainly lymphoplasmacellular inflammation (Figure 3) with or without neutrophilic inflammation, without fibrosis], chronic lesions [fibrosis (Figures 4 and 5) with or without lymphoplasmacellular inflammation, without neutrophilic inflammation], and chronic active lesions (neutrophilic inflammation combined with fibrosis and often lymphoplasmacellular inflammation). A few cases showing both features of acute and subacute lesions were classified as acute/ subacute. The clinical scoring of the severity of the UCD lesions was compared with the histopathological findings.

\section{Statistical Analysis}

To estimate whether the numbers of positive samples specific for a bacterial species differed significantly be- 


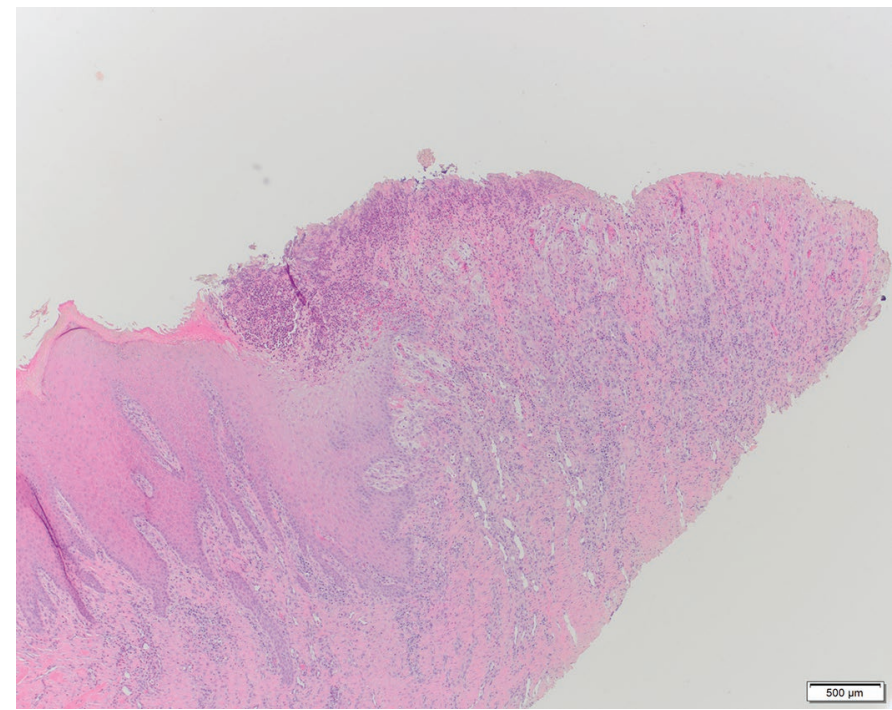

Figure 1. Histopathological features seen in udder cleft dermatitis lesions. Routine hematoxylin and eosin stain, 40×. Ulceration of the skin, with neutrophilic inflammation.

tween UCD categories (healthy, mild UCD, or severe UCD) a 2-sample proportion test (Statistix 8, 2005; https:/www.statistix.com) was used. Only bacterial species that were found in at least 4 samples and in a proportion of one-sixth in at least 1 UCD category were evaluated. $P$-values lower than 0.05 were regarded as significant. Other comparisons (for instance, differences between distributions of bacteria) were descriptive.

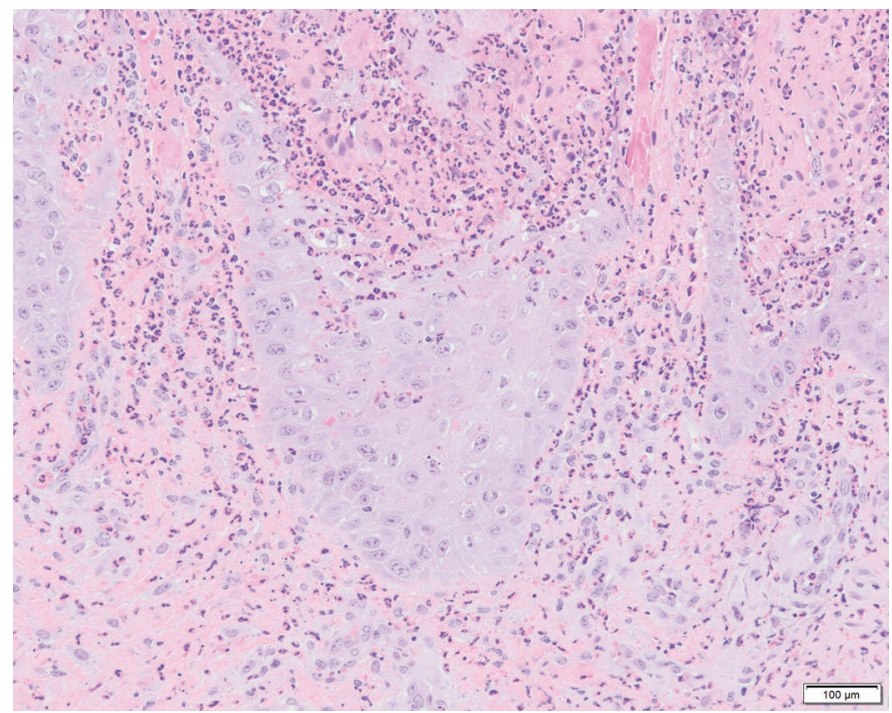

Figure 2. Histopathological features seen in udder cleft dermatitis lesions. Routine hematoxylin and eosin stain, 200×. Neutrophilic inflammation, in this case within both the epidermis and the superficial dermis.

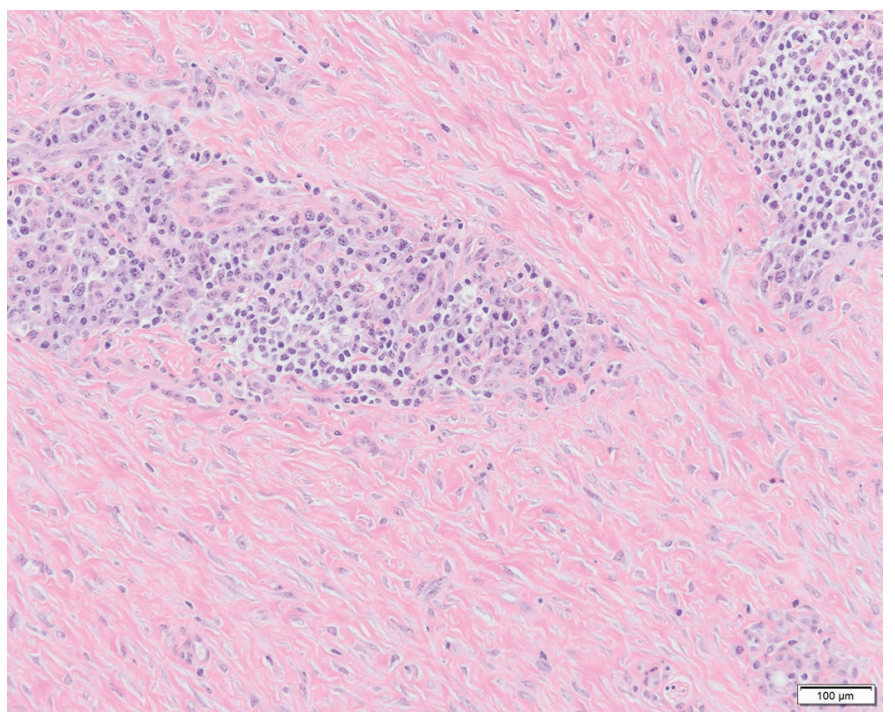

Figure 3. Histopathological features seen in udder cleft dermatitis lesions. Routine hematoxylin and eosin stain, 200x. Lymphoplasmacellular inflammation perivascular with, in this case, a few additional neutrophils and dermal fibrosis.

\section{RESULTS}

\section{Bacteriological Culture}

Deep Samples. In the series of 87 deep-layer samples, T. pyogenes was more often detected in severe UCD lesions compared with mild UCD lesions or healthy skin $(P<0.01)$. As shown in Table 2, Staph. aureus and E. coli were isolated infrequently (6 and

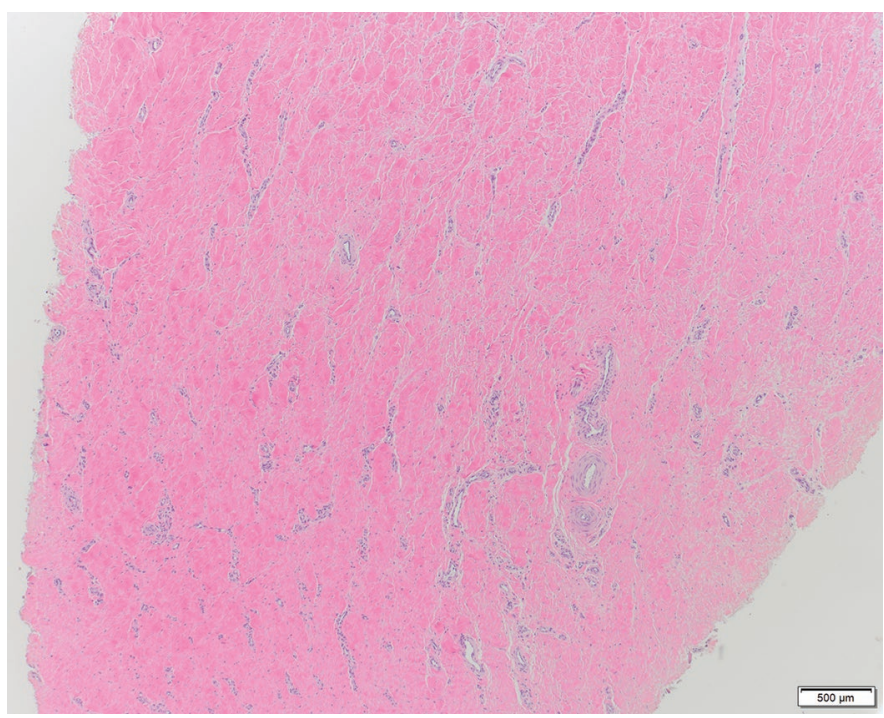

Figure 4. Histopathological features seen in udder cleft dermatitis lesions. Routine hematoxylin and eosin stain, 40×. Fibrosis of the dermis and the subcutaneous tissue, characteristic of chronic and chronic active lesions. 


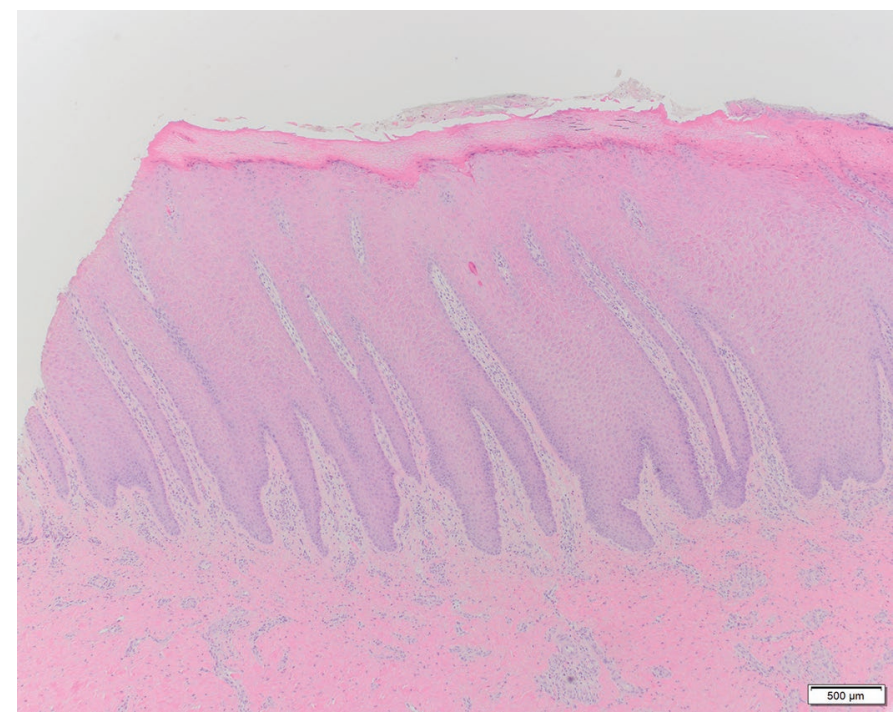

Figure 5. Histopathological features seen in udder cleft dermatitis lesions. Routine hematoxylin and eosin stain, 40×. Epidermal hyperplasia, epidermal hyperkeratosis, and dermal fibrosis.

5 times respectively). Several Clostridium species were identified after anaerobic culture (Table 1). Clostridium sporogenes was found only in animals with mild UCD lesions or healthy skin and not in severe lesions $(P<$ 0.05), whereas Clostridium perfringens was found in all UCD categories $(P>0.05)$.

After anaerobic culture on specific medium, several gram-negative strict anaerobic bacteria were found. A large proportion of the bacteria could be identified via MALDI-TOF. The most commonly found bacterial species was Bacteroides pyogenes. This species was found more often in severe UCD lesions compared with mild UCD lesions or healthy skin $(P<0.01)$.

At culture of 22 samples with severe UCD on Sabouraud plates, fungi were found in 2 cases and yeasts in 4 cases.

Superficial Samples. In a total of 15 swab samples taken from live dairy cows, after aerobic culture T. pyogenes prevalence differed $(P<0.05)$ between cows with severe UCD and the other groups. No E. coli or Staph. aureus colonies were seen. The nonselective culture under anaerobic conditions did not show growth of other bacteria. The anaerobic culture enriched with specific gram-negative supplement, however, showed growth of several species of bacteria (Table 3). Bacteroides pyogenes was more often $(P<0.05)$ present in samples from cows with severe UCD compared with cows with healthy skin. Fungi were found only in low numbers in 1 severe UCD lesion.

\section{Histopathology}

The clinically healthy skin samples $(\mathrm{n}=44)$ did not show significant histopathological abnormalities except in 1 case. Additionally, 15 out of 42 tissue samples from cows with mild UCD lesions did not show histopathological abnormalities (Table 4).

In the other cases of mild UCD and in cases of severe UCD, the lesions were categorized as acute, acute/subacute, chronic, or chronic active, based on the following

Table 2. Numbers of deep-layer tissue samples, categorized as healthy skin or mild or severe udder cleft dermatitis (UCD), in which bacterial species were cultured via aerobic or anaerobic cultures or anaerobic culture on Columbia sheep blood agar with gram-negative supplement (CSGN)

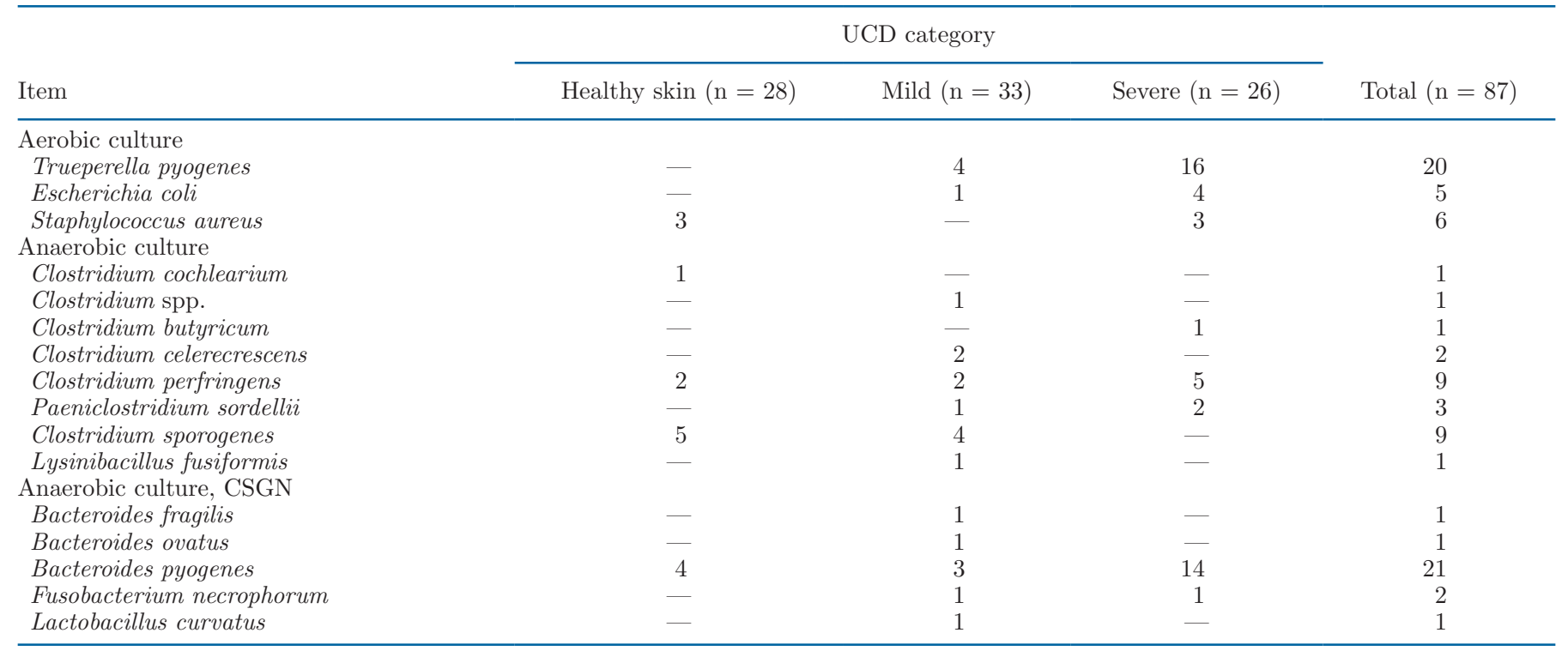


Table 3. Numbers of swab samples of live dairy cows, categorized as healthy skin or mild and severe udder cleft dermatitis (UCD), in which bacterial species were cultured via aerobic culture or anaerobic culture on Columbia sheep blood agar with gram-negative supplement (CSGN)

\begin{tabular}{|c|c|c|c|c|}
\hline \multirow[b]{2}{*}{ Item } & \multicolumn{3}{|c|}{ UCD category } & \multirow[b]{2}{*}{ Total $(\mathrm{n}=5)$} \\
\hline & Healthy skin $(\mathrm{n}=5)$ & Mild $(\mathrm{n}=5)$ & Severe $(\mathrm{n}=5)$ & \\
\hline \multicolumn{5}{|l|}{ Aerobic culture $^{1}$} \\
\hline Trueperella. pyogenes & - & - & 4 & 4 \\
\hline Escherichia coli & - & - & - & - \\
\hline Staphylococcus aureus & - & - & - & - \\
\hline \multicolumn{5}{|l|}{ Anaerobic culture on CSGN } \\
\hline Fusobacterium gonidiaformans & - & - & 2 & 2 \\
\hline Fusobacterium necrophorum & - & - & 2 & 2 \\
\hline Lactobacillus brevis & - & 1 & - & 1 \\
\hline
\end{tabular}

${ }^{1}$ Anaerobic culture using standard medium had the same results as the aerobic culture.

features in variable combination: ulceration, epidermal hyperplasia and epidermal hyperkeratosis, mainly lymphoplasmacellular inflammation, mainly neutrophilic inflammation, and fibrosis. The majority of the severe UCD cases (36 of 41) showed histopathological abnormalities classified as chronic or chronic active, and clinically mild UCD cases showed this inflammatory pattern in only about half of the cases (22 of $42 ; P<$ 0.05). In particular, the chronic active inflammatory pattern was found almost exclusively in severe cases (17 of 18).

Only 8 animals out of 128 showed an acute, acute/ subacute, or subacute histopathological pattern. Clinically 5 of these were mild UCD lesions, and 3 were severe.

The HE routine stain and the additional periodic acid Schiff and Wharthin-Starry stains did not demonstrate fungi or spirochaetes in the tissue. Yeasts were found at the surface of the skin in 2 mild UCD cases. In 1 case the yeast was present in the infundibular part of the hair follicles, which is not consistent with only contamination. In the other case, low yeast numbers were present at the skin surface in absence of skin lesions. In both the HE and Brown-Brenn stains, large numbers of bacteria were present on the skin surface and in the

Table 4. Histopathological categorization of the character of udder cleft dermatitis lesions (rows) versus the clinical severity score of the same lesions (columns)

\begin{tabular}{lcrrr}
\hline Item & $\begin{array}{c}\text { Healthy } \\
\text { skin }\end{array}$ & Mild & Severe & Total \\
\hline Normal & 44 & 15 & 2 & 61 \\
Acute & - & 1 & 1 & 2 \\
Acute/subacute & - & 3 & 1 & 4 \\
Subacute & - & 1 & 1 & 2 \\
Chronic & - & 21 & 18 & 40 \\
Chronic active & 45 & 1 & 18 & 19 \\
Total & 42 & 41 & 128 \\
\hline
\end{tabular}

lateral margins of the tissue samples, consistent with contamination of the lateral margins by taking the samples. Bacteria were also seen within deep dermal or subcutaneous foci, with acute necrosis and necrosuppurative inflammation in a few of the acute to subacute cases. However, in all other cases, the bacteria were present only in the superficial crusts or the superficial necrotic tissue in areas with ulceration, consistent with contamination.

\section{DISCUSSION}

In this study, we showed the presence of some specific anaerobic bacteria species within UCD lesions, as far as possible excluding contamination by taking deep samples after scalding. We found T. pyogenes more often in severe lesions compared with mild lesions or healthy skin. This is in line with the findings of Sorge et al. (2019), who also found increased presence of Trueperella species, although not determined on species level, and Millar et al. (2017), who found these bacteria in embolic lung lesions. Sorge et al. (2019) also found a higher presence of anaerobic bacteria such as Fusobacterium, Helcococcus, Anaerococcus, Porphyromonas, Prevotella, and Bacteroides compared with control skin samples. From these genera we found B. pyogenes with increased frequency in severe UCD. One might suggest that in our study, the other genera could have been missed due to difficulties in culturing these bacteria because of their anaerobic nature. In our opinion, however, this effect is limited, because we have previously cultured Fusobacterium, Helcococcus, Porphyromonas, and Prevotella (Van Engelen et al., 2016) in our diagnostics laboratory. In general, UCD lesions appear to be oxygen depleted and characterized by mixed anaerobic flora in which pyogenic bacteria are dominant and other species might complicate the course of this disease. A review study in humans proved that strict 
anaerobic bacteria were important in chronic wounds (Brook, 2016). Comparing molecular biology with aerobic culture in the wounds showed that relevant flora were missed with culture. This effect would have been diminished if culturing was performed strictly anaerobically (Rhoads et al., 2012). Several clostridial species were cultured from the UCD lesions. From these species, especially $P$. sordellii is of pathogenic importance in the case of wound infections, however, we found no correlation between the severity of the lesions and the presence of Paeniclostridium sordellii. Fulminant clinical signs, such as gas gangrene (Uzal et al., 2016), were not observed in our cows with UCD lesions, and the histopathological features were not consistent with gas gangrene; therefore, we do not expect clostridia to have a causative role in UCD.

In both the HE and Brown-Brenn stains, large numbers of bacteria were present on the skin surface and in the lateral margins of the tissue samples. This was obviously due to the fact that these samples were taken without prior disinfection in a contaminated environment. However, in a few cases, bacteria were also seen within lesions deep in the tissue. These bacteria could be the species that were identified after culture and might be of clinical relevance.

Based on literature about skin lesions in other animals (Foster, 2012) and humans (Howell-Jones et al., 2005), we initially expected that we might culture bacteria such as Staph. aureus or T. pyogenes, or E. coli due to fecal contamination. However, Enterobacteriaceae such as E. coli and staphylococci such as Staph. aureus were not cultured frequently from the lesions. For Staph. aureus, this is in line with the findings of Sorge et al. (2019). Staphylococcus aureus is a common bovine pathogen, residing in udder tissue and belonging to the resident skin flora. In other animal species and humans, Staph. aureus is a common cause of dermatitis, but in bovines, this is not the case. This might be due to specific characteristics of Staph. aureus strains in bovines, as they belong mostly to ruminant-associated clonal complexes and differ from strains found in other animals (Bar-Gal et al., 2015).

We could not find evidence for the involvement of mites, fungi, or treponemes. Mites or any histopathological or clinical indication of mange were not found. Fungi were cultured in a few cases but not found via histopathology, suggesting contamination or fungal presence only within the crusts in direct contact with the environment. Treponemes were not found via histopathology, including in the Wharthin-Starry stain. The absence of these potential causes is in contrast with the findings of some other studies (Warnick et al., 2002; Stamm et al., 2009; Sobhy et al., 2019). Our findings are in line with the study of Sorge et al. (2019), in which also no involvement of treponemes or mites could be demonstrated.

It could be suggested that not detecting specific bacterial species by culture was due to the limitations of the methods used. One might suggest that the animals could have been sampled in more than one location. Even more important, for a positive bacterial culturing, bacteria need to be viable. Purulent suppurative inflammation is commonly found to be culture negative, due to the toxic character of that environment, yet bacteria are thought to initiate the inflammatory process. Additionally, culture conditions must be sufficient for this particular bacterial species, and the culture of strict anaerobic bacteria can be demanding. Nevertheless, we did culture these bacteria, and we performed histopathology of the lesions. For treponemes Sorge et al. (2019) reported the same negative results using molecular methods that did not have the limitations of culturing. In our opinion, culturing methods and molecular methods are complementary. Culturing can be highly sensitive compared with molecular biology, but molecular methods enable the detection of DNA of dead bacteria. It is our opinion that these findings together give ample evidence that treponemes do not play a role in existing UCD lesions.

A discrepancy was found between the clinical severity of the UCD lesions and the histopathological findings. Two cases with severe clinical UCD lesions did not show histopathological abnormalities. This was probably due to nonrepresentative tissue samples caused by sampling the tissue at the periphery of the lesions. The histopathological findings in themselves are aspecific and can be seen in the different phases of healing ulcerative lesions, but they give information concerning the duration of the lesions and, in the case of chronic active lesions, indicate persistence of the cause (McGavin and Zachary, 2007).

In our study, udder cleft dermatitis lesions were histopathologically often characterized by a chronic or chronic active inflammation. This chronic character is in line with the studies of Bouma et al. (2016) and van Werven et al. (2018). This last study showed that even intensive treatment for a long period had limited results. Animals with severe UCD more often showed chronic active inflammation, compared with those with mild UCD. Chronic active inflammation occurs when the inciting stimulus remains present in the chronic stage of the inflammation and continues to elicit an acute inflammatory response (McGavin and Zachary, 2007 , p. 165). In the case of UCD lesions, the inflammation could be actively maintained by the presence of anaerobic bacteria or by the nonhealing character of the skin lesions, which enables bacteria to breach the skin barrier. 
Chronic lesions need proper wound bed preparation, including appropriate cleansing and debridement practices. Removal of debris and necrotic material will improve host defense mechanisms and contribute to better wound healing (Totty et al., 2017).

\section{CONCLUSIONS}

In our study, we aimed to assess the involvement of bacteria, fungi, and parasites in UCD lesions and related this to histopathology of the lesions and clinical scoring. Cultures showed the presence of anaerobic bacteria. Bacteroides pyogenes and T. pyogenes were related to severe lesions, which often had a chronic active character. Other pathogens that have previously been suggested as playing a role in UCD, such as $E$. coli, Staph. aureus, treponemes, fungi, and mites, were not shown to be involved. For the future, the anaerobic character of the bacterial infection and its possible effect on delayed wound healing should be taken seriously. Preventing mild UCD lesions from becoming severe, by inspection of the ventral abdominal wall of cows on a regular basis, followed by effective treatment, will diminish the proportion of anaerobic bacteria and prevent the lesion from becoming chronic.

\section{ACKNOWLEDGMENTS}

We thank the Dutch Dairy Board (Zoetermeer, the Netherlands) for funding this study. The authors declare that they have no conflicts of interest.

\section{REFERENCES}

Bar-Gal, G. K., S. E. Blum, L. Hadas, R. Ehricht, S. Monecke, and G. Leitner. 2015. Host-specificity of Staphylococcus aureus causing intramammary infections in dairy animals assessed by genotyping and virulence genes. Vet. Microbiol. 176:143-154. https://doi.org/ 10.1016/j.vetmic.2015.01.007.

Bouma, A., M. Nielen, E. van Soest, S. Sietsma, J. van den Broek, T. Dijkstra, and T. van Werven. 2016. Longitudinal study of udder cleft dermatitis in 5 Dutch dairy cattle herds. J. Dairy Sci. 99:4487-4495.

Brook, I. 2016. Spectrum and treatment of anaerobic infections. J. Infect. Chemother. 22:1-13. https://doi.org/10.1016/j.jiac.2015.10 .010 .

Ekman, L., A. K. Nyman, H. Landin, U. Magnusson, and K. P. Waller. 2018. Mild and severe udder cleft dermatitis - Prevalence and risk factors in Swedish dairy herds. J. Dairy Sci. 101:556-571. https:// doi.org/10.3168/jds.2017-13133.

Evans, N. J., D. Timofte, S. D. Carter, J. M. Brown, R. Scholey, D. H. Read, and R. W. Blowey. 2010. Association of treponemes with bovine ulcerative mammary dermatitis. Vet. Rec. 166:532-533. https: //doi.org/10.1136/vr.b4822.

Foster, A. P. 2012. Staphylococcal skin disease in livestock. Vet. Dermatol. 23:342-351. https://doi.org/10.1111/j.1365-3164.2012 .01093.x.

Grice, E. A., and J. A. Segre. 2011. The skin microbiome. Nat. Rev. Microbiol. 9:244-253. https://doi.org/10.1038/nrmicro2537.
Howell-Jones, R.S., M.J. Wilson, K.E. Hill, A.J. Howard, P.E. Price, and D.W. Thomas. 2005. A review of the microbiology, antibiotic usage and resistance in chronic skin wounds. J. Antimicrob. Chemother. 55:143-149.

Kallstrom, G. 2014. Are quantitative bacterial wound cultures useful? J. Clin. Microbiol. 52:2753-2756. https://doi.org/10.1128/JCM .00522-14.

McGavin, M. D., and J. F. Zachary. 2007. Pathologic Basis of Veterinary Disease. 4th ed. Mosby-Elsevier, St. Louis, MO.

Millar, M., A. Foster, J. Bradshaw, A. Turner, R. Blowey, N. Evans, and G. Hateley. 2017. Embolic pneumonia in adult dairy cattle associated with udder cleft dermatitis. Vet. Rec. 180:205-206. https: //doi.org/10.1136/vr.j954.

Olde Riekerink, R. G., K. Van Amersfort, O. C. Sampimon, G. A. Hooijer, and T. J. Lam. 2014. Prevalence, risk factors, and a field scoring system for udder cleft dermatitis in Dutch dairy herds. J. Dairy Sci. 97:5007-5011. https://doi.org/10.3168/jds.2013-7651.

Rhoads, D. D., R. D. Wolcott, Y. Sun, and S. E. Dowd. 2012. Comparison of culture and molecular identification of bacteria in chronic wounds. Int. J. Mol. Sci. 13:2535-2550. https://doi.org/10.3390/ ijms13032535.

Scherpenzeel, C. G. M., N. Meertens, and K. Peperkamp. 2020. Gecompliceerde Udder Cleft Dermatitis bij Nederlandse melkkoeien van verschillende bedrijven [Complicated udder cleft dermatitis in Dutch dairy cows from different farms]. Tijdschr. Diergeneeskd. 145:40-43.

Sobhy, N. M., Y. S. Mahmmod, W. Refaai, and A. Awad. 2019. Molecular detection of Treponema species organisms in foremilk and udder cleft skin of dairy cows with digital dermatitis. Trop. Anim. Health Prod. https://doi.org/10.1007/s11250-019-02072-0.

Sorge, U. S., E. M. Binger, J. Schefers, and P. J. Plummer. 2019. Short communication: Metagenomic evaluation of skin biopsies of udder sores in dairy cows. J. Dairy Sci. 102:11470-11475. https://doi .org/10.3168/jds.2018-15863.

Stamm, L. V., R. L. Walker, and D. H. Read. 2009. Genetic diversity of bovine ulcerative mammary dermatitis-associated Treponema. Vet. Microbiol. 136:192-196. https://doi.org/10.1016/j.vetmic .2008.10.022.

Suvarna, S. K., C. Layton, and J. D. Bancroft. 2019. Bancroft's Theory and Practice of Histological Techniques. 8th ed. Elsevier, Amsterdam, the Netherlands. https://doi.org/10.1016/C2015-0-00143-5.

Totty, J. P., N. Bua, G. E. Smith, A. E. Harwood, D. Carradice, T. Wallace, and I. C. Chetter. 2017. Dialkylcarbamoyl chloride (DACC)-coated dressings in the management and prevention of wound infection: A systematic review. J. Wound Care 26:107-114. https://doi.org/10.12968/jowc.2017.26.3.107.

Uzal, A. F., J. G. Songer, J. F. Prescott, and M. R. Popoff. 2016. Clostridial Diseases of Animals. 1st ed. John Wiley and Sons Inc., Ames, IA.

Van Engelen, E., M. Gonggrijp, M. Holzhauer, A. Ten Wolthuis, and A. Velthuis. 2016. Prevalence, risk factors and bacterial species associated with non-healing white line disorders. Abstract. 16th International Conference on Production Diseases, Wageningen, the Netherlands. Wageningen Academic Publishers, Wageningen, the Netherlands. https://doi.org/10.3920/978-90-8686-831-5.

van Werven, T., J. Wilmink, S. Sietsma, J. van den Broek, and M. Nielen. 2018. A randomized clinical trial of topical treatments for mild and severe udder cleft dermatitis in Dutch dairy cows. J. Dairy Sci. 101:8259-8268. https://doi.org/10.3168/jds.2017-13778.

Warnick, L. D., D. Nydam, A. Maciel, C. L. Guard, and S. E. Wade. 2002. Udder cleft dermatitis and sarcoptic mange in a dairy herd. J. Am. Vet. Med. Assoc. 221:273-276. https://doi.org/10.2460/ javma.2002.221.273.

\section{ORCIDS}

E. van Engelen () https://orcid.org/0000-0001-9941-1130

N. M. Meertens (๑ https://orcid.org/0000-0001-9265-413X

T. van Werven $\odot$ https://orcid.org/0000-0003-2391-0253 